\title{
Congenital Anomalies of the Kidney and Urinary Tract in Children with Congenital Heart Defects
}

\author{
Dapeng Jiang Qi Wang Zhengzhou Shi Jie Sun \\ Department of Urology, Shanghai Children's Medical Center, Shanghai Jiao Tong University \\ School of Medicine, Shanghai, China
}

\section{Keywords}

Congenital heart defect - Congenital kidney and urinary tract malformation - Prevalence .

Ultrasonography · Hydronephrosis

\begin{abstract}
Background/Aims: To investigate the incidence and clinical characteristics of congenital anomalies of the kidney and urinary tract (CAKUT) in children with congenital heart defects (CHD). Methods: We retrospectively analyzed the clinical data of children with CHD with CAKUT admitted to the Shanghai Children's Medical Center affiliated with the Shanghai Jiao Tong University School of Medicine between September 2018 and March 2019. Patients underwent routine examinations for liver, kidney, and coagulation function, and urinary tract ultrasonography, and we summarized patients' clinical manifestations and imaging abnormalities. Results: A total of 1,410 children with CHD were diagnosed and treated in our hospital. The total number of patients with abnormal urogenital systems was 104, and hydronephrosis was the most common abnormality, followed by vesicoureteral reflux and duplication of the kidney and ureter. The overall prevalence of CAKUT was $7.4 \%$. There was no statistically significant difference for maternal age, sex, parity, gestational age, and history of medication during pregnancy between the patients with CAKUT and those without CAKUT. Conclusion: The incidence of CAKUT in our patients with CHD was significantly higher than that in the general population. We recommend urinary ultrasonography as a routine examination for children with CHD for early detection of CAKUT, to avoid missed diagnoses, and to initiate appropriate treatment.




\section{Kidney \\ Blood Pressure \\ Research}

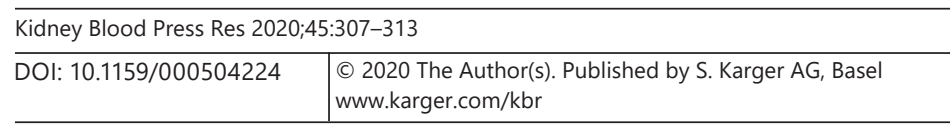

Jiang et al.: CAKUT and CHD in Children

\section{Introduction}

Previous studies in children with congenital anomalies of the kidney and urinary tract (CAKUT) have shown that congenital heart defects (CHD) are the most important comorbidity [1-3]. Specific genetic factors, environmental influences on fetal development, and medications during pregnancy may form the underlying cause of concurrent CAKUT in CHD $[2,4,5]$. Knowledge of the prevalence, types, maternal risk factors, and the natural history of CAKUT associated with CHD are crucial; however, few studies have addressed this topic. Some researchers have stressed that special effort should be made to understand the pathogenesis of CAKUT $[6,7]$. Assessing the clinical significance of this condition is also necessary. Therefore, we performed this retrospective study not only to investigate the prevalence of CAKUT associated with CHD in patients undergoing surgery; our aim was to present a descriptive analysis of the nature of CAKUT. We also examined the risk factors and follow-up results in a large number of patients with CHD who underwent surgery.

\section{Materials and Methods}

\section{Study Design and Participants}

All patients with CHD underwent surgery at the Department of Cardiac and Thoracic Surgery, Shanghai Children's Medical Center, Shanghai Jiao Tong University School of Medicine between September 2018 and March 2019. Data for patients aged $<12$ years (age at surgery) with CHD were retrospectively evaluated and were screened for the presence of CAKUT. Ultrasonographic parameters and clinical information (including prenatal consultation records) were obtained from all available electronic medical record databases. Patients without available information for the ultrasonographic parameters were excluded from the analysis. Patients with Society for Fetal Urology grade 1 hydronephrosis were also excluded. Patent foramen ovale and patent ductus arteriosus were not considered as CHD in newborns.

The following clinical and demographic data were collected for each patient: age, gestational age, sex, family history (CHD, CAKUT, or other congenital anomalies), type and side of CAKUT, type of CHD, history of urinary tract infection, time since CKD diagnosis, time since CAKUT diagnosis, and other associated anomalies. CAKUT was defined by renal ultrasonography in all patients and, on indication, by voiding cystourethrogram and/or radionuclide evaluation and magnetic resonance urography. CAKUT variants of concern were: renal agenesis, renal dysplasia/hypoplasia, hydronephrosis (ureteropelvic junction obstruction or ureterovesical junction obstruction), vesicoureteral reflux (VUR), duplex collecting system, horseshoe kidney, and posterior urethral valves. The mean follow-up period was 11 months (range 6-24) after surgery for CHD.

\section{Statistical Analysis}

Data are shown as mean \pm SD for continuous data and percentage for categorical variables. The 2-tailed unpaired $t$ test was used to determine statistically significant differences in variables. The Fisher exact test was used to compare proportions. All $p$ values of $<0.05$ were accepted as statistically significant. 
Kidney

Blood Pressure

Research

Table 1. The frequency of each type of CHD and CAKUT

\begin{tabular}{l|l}
\hline Kidney Blood Press Res 2020;45:307-313 \\
\hline DOI: 10.1159/000504224 & $\begin{array}{l}\text { (c) 2020 The Author(s). Published by S. Karger AG, Basel } \\
\text { www.karger.com/kbr }\end{array}$ \\
\hline
\end{tabular}

Jiang et al.: CAKUT and CHD in Children

\begin{tabular}{lcc}
\hline Type of CHD & $n(\%)$ & $\begin{array}{l}\text { CHD+CAKUT } \\
n\left(\%: \mathrm{n} / \mathrm{n}_{\mathrm{CHD}}\right)\end{array}$ \\
\hline ASD & $494(35)$ & $39(7.9)$ \\
VSD & $462(32.8)$ & $33(7.1)$ \\
PDA & $87(6.2)$ & $7(8)$ \\
TOF & $41(2.92)$ & $4(9.8)$ \\
AVSD & $39(2.7)$ & $3(7.95)$ \\
PS & $37(2.6)$ & $2(5.4)$ \\
PVC & $37(2.6)$ & $3(8.1)$ \\
HLHS & $32(2.3)$ & $2(6.3)$ \\
PA & $29(2.1)$ & $1(3.45)$ \\
TGA & $11(0.78)$ & $0(0)$ \\
Others & $141(10)$ & $10(7.1)$ \\
\hline
\end{tabular}

ASD, atrial septal defect; AVSD, atrioventricular septal defect; CAKUT, congenital anomalies of the kidney and urinary tract; CHD, congenital heart defects; HLHS, hypoplastic left heart syndrome; PA, pulmonary atresia; PDA, patent ductus arteriosus; PS, pulmonary stenosis; PVC, pulmonary venous connection; TGA, transposition of great arteries; TOF, tetralogy of fallot; VSD, ventricular septal defect.

\begin{tabular}{lc}
\hline Feature & \\
\hline Mean age at surgery, months \pm SD & $17 \pm 21.4$ \\
Sex ratio (M:F ratio) & $749: 661(1.13: 1)$ \\
Prevalence of CAKUT, $n(\%)$ & $104(7.4)$ \\
1. Hydronephrosis & 41 \\
2. VUR & 26 \\
3. Duplicated collecting duct systems & 18 \\
Prevalence of additional urogenital & \\
$\quad$ findings, $n(\%)$ & $39(2.85)$ \\
Other associated malformations, $n(\%)$ & $160(12.3)$ \\
1. Musculoskeletal anomalies & 71 \\
2. Digestive system anomalies & 47 \\
3. Ear, face, and neck anomalies & 42
\end{tabular}

CAKUT, congenital anomalies of the kidney and urinary tract; CHD, congenital heart defects; VUR, vesicoureteral reflux.
Table 2. Demographic and clinical characteristics of CHD patients 
Table 3. Demographic characteristics of CHD patients with CAKUT

\begin{tabular}{llll}
\hline Feature & $\begin{array}{l}\text { CHD without CAKUT } \\
(n=1,306)\end{array}$ & $\begin{array}{l}\text { CHD + CAKUT } \\
(n=104)\end{array}$ & $p$ \\
\hline Mean age at surgery, months \pm SD & $16.5 \pm 20.1$ & $18.2 \pm 22.3$ & 0.333 \\
Gestational age, weeks \pm SD & $37.9 \pm 4$ & $38.2 \pm 5$ & 0.056 \\
Sex ratio (M:F ratio) & $1.15: 1$ & $0.93: 1$ & 0.284 \\
Maternal age, years \pm SD & $28.3 \pm 9.3$ & $27.6 \pm 6.2$ & 0.03 \\
Birth weight, kg \pm SD & $3.16 \pm 0.55$ & $2.86 \pm 0.6$ & $<0.0001$ \\
Prevalence of additional urogenital findings, $n(\%)$ & $35(2.7)$ & $4(3.8)$ & 0.484 \\
Other associated malformations, $n(\%)$ & $140(10.7)$ & $20(19.23)$ & 0.008 \\
\hline
\end{tabular}

CAKUT, congenital anomalies of the kidney and urinary tract; CHD, congenital heart defects.

(41 patients). VUR was diagnosed in 26 patients (Table 2). High-grade (III-V) VUR was present in 6 patients $(23.1 \%)$.

There was no correlation between CHD type and the different CAKUT variants. Of the patients with CAKUT, there is almost no difference in the prevalence between girls and boys. (54/104 for girls vs. 50/104 for boys; $p>0.05$ ). Serum blood urea nitrogen and creatinine levels were normal in all patients with CAKUT. Additional urogenital findings other than CAKUT were detected in 39 patients (cryptorchidism in 20, hypospadias in 18, and ovarian teratoma in 1). Other comorbidities were present in $19.2 \%$ of the patients with CAKUT. Among the 1,306 patients without urogenital anomalies, other comorbidities were present in $10.7 \%$ of the patients. The most frequent anomalies were musculoskeletal anomalies, digestive system anomalies, and ear, face, and neck anomalies.

The maternal age of mothers of patients with CAKUT versus mothers of unaffected children did not differ. Children with CAKUT had a significantly lower birth weight compared with children without CAKUT (2.89 vs. $3.16 \mathrm{~kg}$, respectively; $p<0.001$ ) (Table 3). A total of 62 of 104 patients with CAKUT were diagnosed prenatally, and $59.6 \%$ of these fetuses had multiple congenital anomalies. Thirty-five cases with hydronephrosis had been diagnosed prenatally. Of these 35 patients, 18 (51.4\%) had resolution before the time of heart surgery. So, the real number of patients with CAKUT is 86 at the time of heart surgery. No abnormality was found in 42 CAKUT patients during prenatal ultrasound examination. Among these 42 children who received no prenatal diagnosis, there were 24 VUR, 9 duplicated collecting duct systems, 2 posterior urethral valves, 1 horseshoe kidney, and 6 hydronephroses.

Eight patients $(7.7 \%)$ underwent renal or ureteral surgery during the follow-up (2 cases with hydronephrosis received pyeloplasty when differential renal function was less than $40 \%$; 2 cases with high-grade [IV-V] VUR received surgery; 2 cases with ectopic upper pole ureters in duplex kidneys were managed surgically by ipsilateral distal ureteroureterostomy; 2 cases with duplicated collecting systems received laparoscopic upper pole nephrectomy because of decreased differential renal function and recurrent pyelonephritis in the upper pole of the duplicated kidney). Moreover, 3 patients among these 8 patients were diagnosed prenatally.

\section{Discussion}

A recent mouse study indicated a significant overlap in the genetic etiology of CHD and congenital kidney abnormalities [2], and may indicate that patients with CHD are at increased risk for complications from renal anomalies. This finding stresses the importance of being 


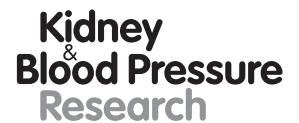

Kidney
Blood Pressure
Research \begin{tabular}{l|l}
\hline Kidney Blood Press Res 2020:45:307-313 \\
\hline DOI: 10.1159/000504224 & $\begin{array}{l}\text { @ 2020 The Author(s). Published by S. Karger AG, Basel } \\
\text { www.karger.com/kbr }\end{array}$ \\
\hline
\end{tabular}

Jiang et al.: CAKUT and CHD in Children

aware of patients with CHD and concurrent CAKUT and the need to explore the related gene mutation(s) [8-10]. However, the clinical characteristics of these patients have not been reported. Our retrospective analysis assessed the prevalence and types of CAKUT associated with CHD in a large pediatric cohort.

The overall prevalence of CAKUT at birth is 3-6 per 1,000 live births [11], and in our study, $7.4 \%$ of children with CHD had concurrent CAKUT. Studies report that the incidence of CAKUT has increased annually [12, 13], which may be secondary to developments and progress in ultrasonography and other imaging technologies that have increased the detection rate. In addition, the compliance of regular pregnancy examinations has increased, which provides a good opportunity for the early detection and treatment of CAKUT.

The most frequent CAKUT variants in our patients were hydronephrosis $(n=41,39.4 \%)$, VUR ( $n=26,25 \%)$, and duplicated collecting duct systems $(n=18,17.3 \%)$. In this series, the most frequent CHD was atrial septal defect $(n=494,35 \%)$, ventricular septal defect $(n=462$, $32.8 \%)$, and patent ductus arteriosus $(n=87,6.2 \%)$. We found no correlation between CAKUT type and cardiac lesion type. These findings are consistent with epidemiological characteristics for structural birth defects in the kidney and urinary tract in the human population and mouse models [2]. Our data also confirmed that CAKUT can occur in patients with CHD with a frequency higher than expected in the normal population. A larger sample size is required to fully identify a genetic effect as causative of this novel association.

Because of the concealed location of the kidneys, ureters, and bladder, anatomical malformations without obvious clinical symptoms during early neonatal development are often missed or ignored by parents. Additionally, symptoms cannot be used as the main diagnostic basis for CAKUT to judge whether there is malformation or aggravation of renal injury. Imaging examination plays an irreplaceable role during follow-up. Studies have found that even in the same group of newborns, the diagnostic rate of CAKUT by postpartum ultrasonography is higher than that by prenatal ultrasonographic screening, confirming that a certain proportion of patients with CAKUT are identified in later stages in children with normal prenatal ultrasonographic findings [14].

Only 3 of our patients were symptomatic. Therefore, children with CHD should be routinely screened by urinary ultrasonography, especially those who were previously found to have subtle abnormalities in the urinary system but were not routinely followed. Ultrasonographic screening is not only of great significance for defining the development of CAKUT, but also can prevent impaired renal function and delay the course of the disease through timely intervention in some serious cases, thus improving the quality of life of children with CAKUT $[15,16]$.

At the time of this report, 8 of 104 children with CAKUT in our study have undergone surgery, and the remainder are still under follow-up observation. The degree of kidney and ureter malformations associated with CAKUT changes over time as does the degree of kidney injury [17]. Therefore, pediatric urologists should perform comprehensive assessment of these children and inform their families of their condition and prognosis to avoid anatomical and functional changes caused by the natural evolution of CAKUT, which otherwise may be misunderstood as surgical complications. CAKUT without indications for surgery has no significant effect on the outcome of CHD surgery, but these patients require follow-up in urology clinics postoperatively. During follow-up, if there are indications for surgery for CAKUT, the operation should be performed as soon as possible.

Because this was a retrospective study, all of our patients with CHD were hospitalized for surgical treatment and to obtain complete urological imaging data. Patients who were not hospitalized and those with missing relevant examination data were not included in our analysis. Therefore, our data do not represent all patients with CHD complicated with CAKUT and its clinical characteristics, nor can we provide a comprehensive understanding of the 
clinical characteristics of CHD combined with CAKUT; this is the main limitation of our study. The accuracy and representativeness of our results will be greatly improved if data for all patients with CHD with CAKUT can be collected.

\section{Conclusion}

In conclusion, the incidence of CAKUT in CHD is significantly higher than that in the general population. Urinary ultrasonography is recommended as a routine examination for children with CHD. Our results indicated that patients with CHD would benefit from routine evaluation of the urinary tract in their diagnostic workup to reduce potential renal complications and improve clinical outcomes. We did not perform gene studies in our patients, so we cannot provide a theoretical basis for defining the cause of CHD complicated with CAKUT at the gene level. This part of the work requires further exploration.

\section{Acknowledgements}

We thank Jane Charbonneau, DVM, from Liwen Bianji, Edanz Group China (www. liwenbianji.cn/ac), for editing the English text of a draft of the manuscript.

\section{Statement of Ethics}

This study was performed in accordance with the principles of the Declaration of Helsinki. The study was approved by the Shanghai Children's Medical Center Research Ethics Committee (SCMCEC -K2019025). Written informed consent was obtained from each participant.

\section{Disclosure Statement}

The authors have no conflicts of interest to declare.

\section{Funding Sources}

This project was supported by a Grant from the Natural Science Foundation of Shanghai (No. 19ZR1432800) and the Program for improving the Clinical Scientific Research ability of Postgraduate students in Pediatrics, School of Medicine, Shanghai Jiaotong University (EKKY2018005DGD).

\section{Author Contributions}

D.J. conceived the concept and contributed to the study design, manuscript writing, and review. Q.W. and Z.S. assisted in conduct of the study and contributed to manuscript writing. J.S. was the principal investigator, contributed to the concept, the study design, conducted the study, drafted the manuscript, and contributed to the review. 


\section{References}

1 Stoll C, Dott B, Alembik Y, Roth MP. Associated nonurinary congenital anomalies among infants with congenital anomalies of kidney and urinary tract (CAKUT). Eur J Med Genet. 2014 Jul;57(7):322-8.

2 San Agustin JT, Klena N, Granath K, Panigrahy A, Stewart E, Devine W, et al. Genetic link between renal birth defects and congenital heart disease. Nat Commun. 2016 Mar;7(1):11103.

3 Schaefer F, Doyon A, Azukaitis K, Bayazit A, Canpolat N, Duzova A, et al.; 4C Study Consortium. Cardiovascular Phenotypes in Children with CKD: The 4C Study. Clin J Am Soc Nephrol. 2017 Jan;12(1):19-28.

4 Li Y, Klena NT, Gabriel GC, Liu X, Kim AJ, Lemke K, et al. Global genetic analysis in mice unveils central role for cilia in congenital heart disease. Nature. 2015 May;521(7553):520-4.

5 Miller A, Riehle-Colarusso T, Alverson CJ, Frías JL, Correa A. Congenital heart defects and major structural noncardiac anomalies, Atlanta, Georgia, 1968 to 2005. J Pediatr. 2011 Jul;159(1):70-78.e2.

6 Jain S, Chen F. Developmental pathology of congenital kidney and urinary tract anomalies. Clin Kidney J. 2018 Dec;12(3):382-99.

7 Katsoufis CP, DeFreitas MJ, Infante JC, Castellan M, Cano T, Safina Vaccaro D, et al. Risk Assessment of Severe Congenital Anomalies of the Kidney and Urinary Tract (CAKUT): A Birth Cohort. Front Pediatr. 2019 May; 7 182.

8 Gabriel GC, Pazour GJ, Lo CW. Congenital Heart Defects and Ciliopathies Associated With Renal Phenotypes. Front Pediatr. 2018 Jun; 6:175.

9 Stoll C, Dott B, Alembik Y, Roth MP. Associated noncardiac congenital anomalies among cases with congenital heart defects. Eur J Med Genet. 2015 Feb;58(2):75-85.

10 Hamadah HK, Hijazi O, Faraji MA, Kabbani MS. Bedside ultrasonography screening for congenital renal anomalies in children with congenital heart diseases undergoing cardiac repair. J Saudi Heart Assoc. 2018 Apr; 30(2):95-101.

11 Harambat J, van Stralen KJ, Kim JJ, Tizard EJ. Epidemiology of chronic kidney disease in children. Pediatr Nephrol. 2012 Mar;27(3):363-73.

12 Macumber I, Schwartz S, Leca N. Maternal obesity is associated with congenital anomalies of the kidney and urinary tract in offspring. Pediatr Nephrol. 2017 Apr;32(4):635-42.

13 Tain YL, Luh H, Lin CY, Hsu CN. Incidence and Risks of Congenital Anomalies of Kidney and Urinary Tract in Newborns: A Population-Based Case-Control Study in Taiwan. Medicine (Baltimore). 2016 Feb; 95(5):e2659.

14 Melo BF, Aguiar MB, Bouzada MC, Aguiar RL, Pereira AK, Paixão GM, et al. Early risk factors for neonatal mortality in CAKUT: analysis of 524 affected newborns. Pediatr Nephrol. 2012 Jun;27(6):965-72.

15 Nef S, Neuhaus TJ, Spartà G, Weitz M, Buder K, Wisser J, et al. Outcome after prenatal diagnosis of congenital anomalies of the kidney and urinary tract. Eur J Pediatr. 2016 May;175(5):667-76.

16 Gong Y, Zhang Y, Shen Q, Xiao L, Zhai Y, Bi Y, et al. Early detection of congenital anomalies of the kidney and urinary tract: cross-sectional results of a community-based screening and referral study in China. BMJ Open. 2018 May;8(5):e020634.

17 Marzuillo P, Guarino S, Grandone A, Di Somma A, Della Vecchia N, Esposito T, et al. Outcomes of a Cohort of Prenatally Diagnosed and Early Enrolled Patients with Congenital Solitary Functioning Kidney. J Urol. 2017 Nov;198(5):1153-8. 